
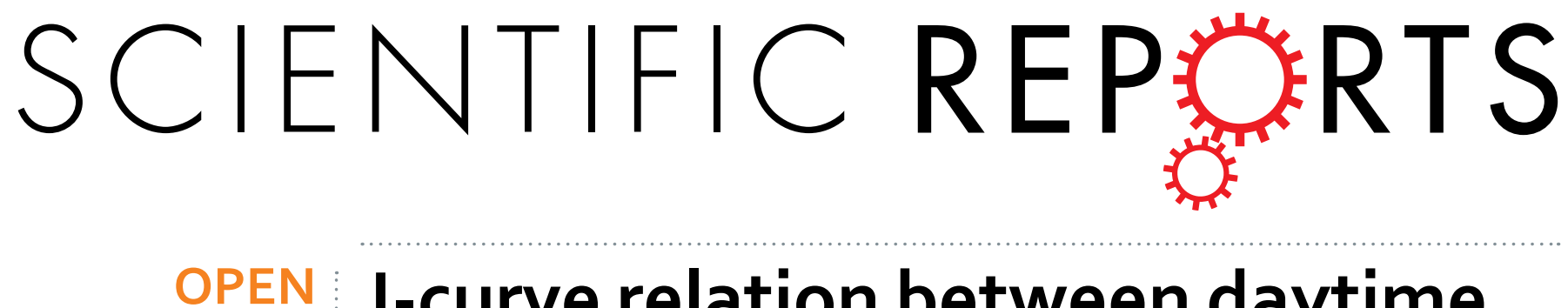

\title{
J-curve relation between daytime nap duration and type 2 diabetes or metabolic syndrome: A dose-
}

Received: 31 May 2016

Accepted: 02 November 2016

Published: 02 December 2016 response meta-analysis

\author{
Tomohide Yamada, Nobuhiro Shojima, Toshimasa Yamauchi \& Takashi Kadowaki
}

Adequate sleep is important for good health, but it is not always easy to achieve because of social factors. Daytime napping is widely prevalent around the world. We performed a meta-analysis to investigate the association between napping (or excessive daytime sleepiness: EDS) and the risk of type 2 diabetes or metabolic syndrome, and to quantify the potential dose-response relation using cubic spline models. Electronic databases were searched for articles published up to 2016, with 288,883 Asian and Western subjects. Pooled analysis revealed that a long nap ( $\geq 60 \mathrm{~min} / \mathrm{day}$ ) and EDS were each significantly associated with an increased risk of type 2 diabetes versus no nap or no EDS (odds ratio $1.46(95 \% \mathrm{Cl} 1.23-1.74, \mathrm{p}<0.01)$ for a long nap and 2.00 (1.58-2.53) for EDS). In contrast, a short nap ( $<60 \mathrm{~min} /$ day) was not associated with diabetes $(p=0.75)$. Dose-response meta-analysis showed a J-curve relation between nap time and the risk of diabetes or metabolic syndrome, with no effect of napping up to about $\mathbf{4 0}$ minutes/day, followed by a sharp increase in risk at longer nap times. In summary, longer napping is associated with an increased risk of metabolic disease. Further studies are needed to confirm the benefit of a short nap.

Adequate sleep is important for maintaining good health, along with a balanced diet and exercise. Several recent studies have shown that the relation between the duration of nocturnal sleep and the risk of type 2 diabetes ${ }^{1,2}$, $\mathrm{CVD}^{3}$, stroke ${ }^{4}$, or all-cause mortality ${ }^{5,6}$ is described by a U-shaped curve. These findings suggest that a moderate amount of sleep, neither too short nor too long, promotes good health. However, it is not always easy to obtain sufficient sleep due to the influence of social factors including work. According to the U.S. Centers for Diseases Control and Prevention, one in three American adults do not get enough sleep ${ }^{7}$.

A nap is defined as a short sleep that is typically taken during daylight hours, and the habit of napping is prevalent worldwide. While a daytime nap is usually brief, it can range from a few minutes to several hours. In addition, the frequency varies from occasional naps to several times daily for habitual nappers. Some people take a nap because of excessive daytime drowsiness resulting from a sleep disorder ${ }^{8}$. Similar to the duration of nighttime sleep, several studies have shown that a long daytime nap is positively correlated with cardiovascular disease ${ }^{9}$ and all-cause mortality ${ }^{10}$.

A short nap $(<30 \mathrm{~min})$ promotes alertness, reduces sleep deficits, and enhances performance and learning ${ }^{11}$. Some epidemiological studies have even suggested that a short nap decreases the risk of cardiovascular disease ${ }^{12}$ and Alzheimer's disease ${ }^{13}$.

We recently published a dose-response meta-analysis that identified a J-curve relationship between nap time and cardiovascular disease based on 1.5 million person-years of data ${ }^{14}$. The relative risk of cardiovascular disease was decreased by a short nap ( 0 to $30 \mathrm{~min}$ ), followed by a sharp increase at longer nap times. In addition, there was a linear relationship between nap time and all-cause mortality.

Recent epidemiological studies on the relation between daytime napping and diabetes or metabolic syndrome have yielded conflicting results ${ }^{15-20}$. However, these studies showed heterogeneity with respect to sample size, stratification of nap times, nocturnal sleep duration, and other characteristics that could have contributed to different outcomes. It is still unclear whether a dose-response relation exists between daytime napping and the risk of diabetes. Therefore, we performed the present meta-analysis to investigate the association between napping or 


\section{Literature search and study selection}
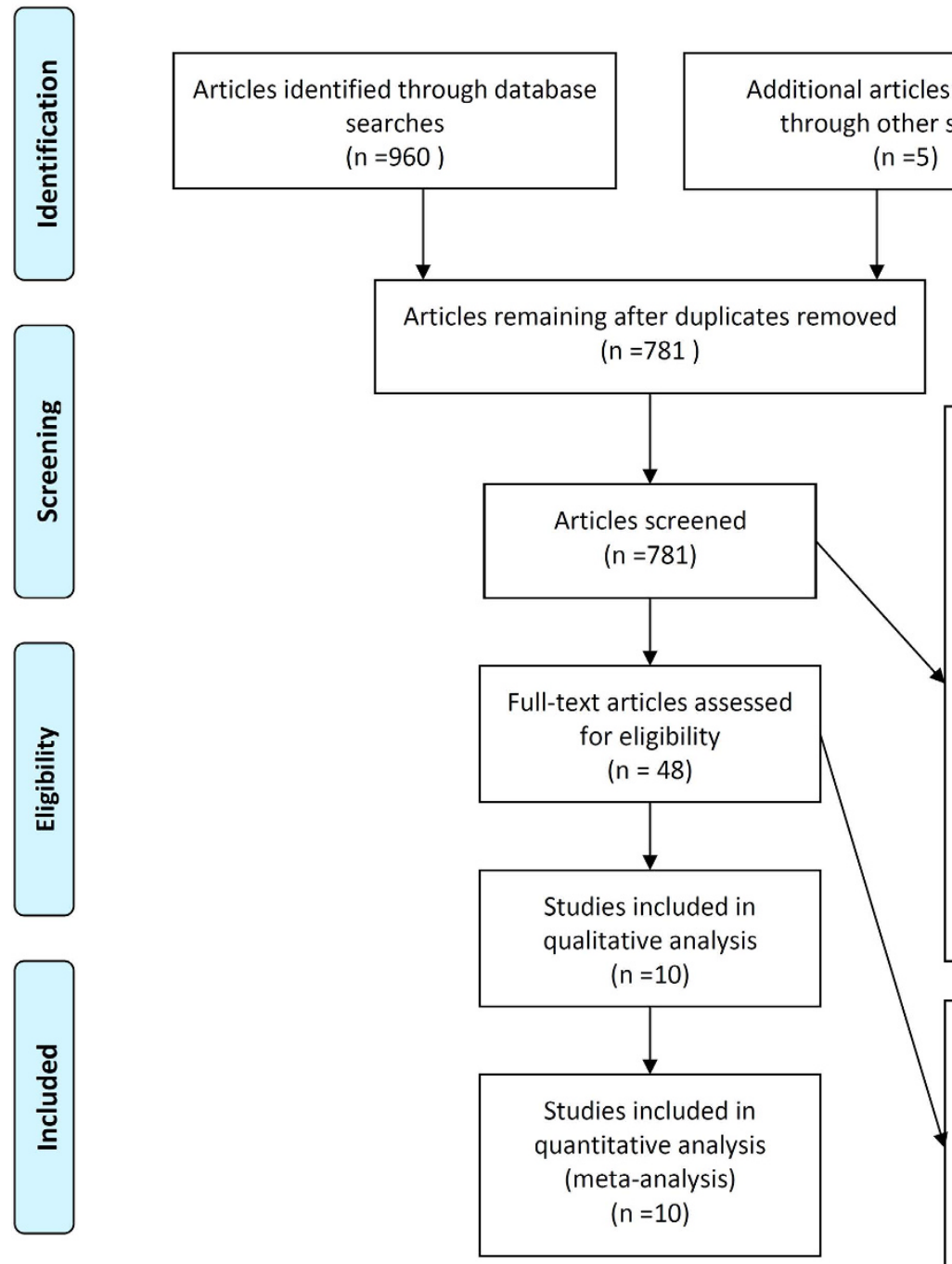

Articles excluded ( $n=733$ ): Reviews, editorials, commentaries. letters, abstracts from scientific meeting, and case reports $(n=251)$, Research not related to sleep, diabetes, or metabolic syndrome ( $n=68$ ), Animal studies and human in vitro and in vivo studies (non-clinical research) $(n=59)$, Irrelevant exposures and outcomes $(n=355)$

Full-text articles excluded ( $n=38)$ : Review ( $n=1)$, Irrelevant exposures and outcomes $(n=20)$, Studies on sleep apnea $(n=14)$, Studies without nap categories $(n=3)$

Figure 1. Literature search and study selection.

excessive daytime sleepiness (EDS) ${ }^{21-24}$ and the risk of diabetes or metabolic syndrome, and we also quantified the potential dose-response relation by using cubic spline models.

\section{Methods}

Data Sources and Searches. We performed a literature search (up to 31 January 2016) of the Medline, Cochrane Library, and Web of Science databases to identify observational studies examining the association between napping and/or excessive daytime sleepiness and the risk of type 2 diabetes and/or metabolic syndrome. The details of the search terms are shown in Table S1. We supplemented this search by performing a manual search of all the references cited in the articles thus identified.

Study selection. We selected studies that reported risk estimates for type 2 diabetes and metabolic syndrome in relation to daytime napping and excessive daytime sleepiness in the general population, and that provided point estimates of odds ratio with the $95 \%$ confidence interval or standard error for qualitative assessment. We performed a combined meta-analysis of the relation between napping and diabetes or excessive daytime sleepiness and diabetes, because napping and daytime sleepiness have overlapping features and are often handled in the same way clinically by physicians and co-medical staff. We defined the presence of daytime napping on the basis of an affirmative answer to questions such as "Do you take a daytime nap?" or "Do you sleep during the day?" Excessive daytime sleepiness was identified by affirmative answers to questions like "Do you have a problem with sleepiness during the daytime?" We excluded studies on type 1 diabetes. The studies that we selected followed the 


\begin{tabular}{|c|c|c|c|c|}
\hline $\begin{array}{l}\text { Author, year of } \\
\text { publication }\end{array}$ & $\begin{array}{l}\text { Study participants location, subject source, and response } \\
\text { rate }\end{array}$ & Assessment of exposure & $\begin{array}{c}\text { Analysis } \\
\text { by sex }\end{array}$ & $\begin{array}{c}\text { Prevalence of } \\
\text { napping (EDS) (\%) }\end{array}$ \\
\hline \multicolumn{5}{|c|}{ Diabetes and Daytime Napping } \\
\hline Stang et al. ${ }^{15}$ a & $\begin{array}{l}\text { Total }=4,458 \text {; diabetes }=355(8.0 \%) ; 52 \% \text { female; age } \\
45-75 \text { years; BMI not available; Germany; Participants } \\
\text { were local residents of industrial cities in the Ruhr region } \\
\text { of Germany. The response rate was } 56 \% \text {. }\end{array}$ & Structured interview & Yes & $\begin{array}{l}\text { Male } 17 \% \text {, female } \\
\quad 15 \%\end{array}$ \\
\hline Xu et al. ${ }^{16 \mathrm{~b}}$ & $\begin{array}{l}\text { Total }=174,020 \text {; diabetes }=10,100(5.8 \%) ; 43 \% \text { female; } \\
\text { age } 62.4 \text { years; BMI } 26.5 \mathrm{~kg} / \mathrm{m}^{2} ; \text { USA; Participants were } \\
\text { members of the American Association of Retired Persons } \\
\text { from six states and two metropolitan areas of the USA. The } \\
\text { response rate was } 56 \% \text {. }\end{array}$ & Self-completed questionnaire & No & $46 \%$ \\
\hline Fang et al. ${ }^{17 \mathrm{c}}$ & $\begin{array}{l}\text { Total }=27,009 ; \text { diabetes }=4,772(17.7 \%) ; 55 \% \text { female; } \\
\text { age } 63.6 \text { years; BMI } 24.5 \mathrm{~kg} / \mathrm{m}^{2} ; \text { China; Participants were } \\
\text { retired employees of Dongfeng Motor Corporation, an } \\
\text { automobile manufacturer in China. The response rate was } \\
87 \% \text {. }\end{array}$ & Self-completed questionnaire & No & $69 \%$ \\
\hline Lam et al. ${ }^{18 \mathrm{~d}}$ & $\begin{array}{l}\text { Total }=19,567 \text { (subsample } 3.822 \text { ); diabetes }=2,642 \\
(13.5 \%) ; 71 \% \text { female; age } 62.2 \text { years; BMI not available; } \\
\text { China; Participants were members of the Guangzhou } \\
\text { Health and Happiness Association for Respectable Elders, } \\
\text { a community social and welfare association. The response } \\
\text { rate was } 90 \% \text { for men and } 99 \% \text { for women. }\end{array}$ & Structured interview & No & $67 \%$ \\
\hline \multicolumn{5}{|c|}{ Diabetes and Excessive Daytime Sleepiness } \\
\hline al. $^{21 e, f}$ & $\begin{array}{l}\text { Total }=6,779 ; \text { diabetes }=\text { not available; } 100 \% \text { female; age } \\
44.7 \text { years; BMI } 24.1 \mathrm{~kg} / \mathrm{m}^{2} ; \text { Sweden; Participants were a } \\
\text { random sample of women living in the city of Uppsala, } \\
\text { Sweden, drawn from the population registry. The response } \\
\text { rate was } 68.9 \% \text {. }\end{array}$ & Self-completed questionnaire & $\begin{array}{c}\text { No } \\
\text { (female } \\
\text { only) }\end{array}$ & $13 \%$ \\
\hline Bixer et al. ${ }^{22 \mathrm{~g}}$ & $\begin{array}{l}\text { Total }=16,583 ; \text { diabetes }=2,156(13 \%) ; 74 \% \text { female; age } \\
46.5 \text { years; BMI } 26.3 \mathrm{~kg} / \mathrm{m}^{2} ; \text { Spain; Participants were a } \\
\text { random sample of } 16,583 \text { men and women from central } \\
\text { Pennsylvania. The response rate was } 73.5 \% \text { (men) and } \\
74.1 \% \text { (women). }\end{array}$ & Structured interview & No & $8.7 \%$ \\
\hline al. $^{23 \mathrm{~h}}$ & $\begin{array}{l}\text { Total }=593 \text {; diabetes }=84(14.3 \%) ; 59 \% \text { female; age } 60 \\
\text { years; BMI not available; Finland; Participants were living } \\
\text { in the City of Oulu in northern Finland. The response rate } \\
\text { was } 71 \% \text {. }\end{array}$ & Self-completed questionnaire & No & $19.6 \%$ \\
\hline Asplund. $^{24 \mathrm{i}}$ & $\begin{array}{l}\text { Total }=6,143 ; \text { diabetes }=\text { not available; } 61 \% \text { female; age } \\
73 \text { years; BMI not available; Sweden; Participants were } \\
\text { members of the National Swedish Pensioners' Association. } \\
\text { The response rate was } 61 \% \text {. }\end{array}$ & Self-completed questionnaire & Yes & $\begin{array}{l}\text { Male } 14 \% \text {, female } \\
\quad 14 \%\end{array}$ \\
\hline \multicolumn{5}{|c|}{ Metabolic Syndrome and Daytime Napping } \\
\hline Wu et al. ${ }^{19 j}$ & $\begin{array}{l}\text { Total }=25,184 ; \text { metabolic syndrome }=8,046(31.9 \%) ; 55 \% \\
\text { female; age } 63.6 \text { years; BMI } 25.8 \mathrm{~kg} / \mathrm{m}^{2} ; \text { China; Participants } \\
\text { were retired employees of Dongfeng Motor Corporation } \\
\text { in Shiyan City, Hubei Province, China. The response rate } \\
\text { was } 87 \% \text {. }\end{array}$ & Self-completed questionnaire & Yes & $\begin{array}{l}\text { Male } 73 \% \text {, female } \\
\quad 65 \%\end{array}$ \\
\hline Lin et al. ${ }^{20 \mathrm{k}}$ & $\begin{array}{l}\text { Total }=8,547 \text {; metabolic syndrome }=3,176(37.2 \%) ; 28.2 \% \\
\text { female; age } 56.0 \text { years; BMI } 23.7 \mathrm{~kg} / \mathrm{m}^{2} ; \text { China; Participants } \\
\text { were from a community in Guangzhou, China. The } \\
\text { response rate was } 98.1 \% \text {. }\end{array}$ & Self-completed questionnaire & Yes & $\begin{array}{l}\text { Male } 56 \% \text {, female } \\
\quad 48 \%\end{array}$ \\
\hline
\end{tabular}

Table 1. Summary of cohort studies evaluating the association between napping and type 2 diabetes. Among these studies, one study (16) was a cohort study with a mean follow-up period of 7-10 years. All of the other studies were cross-sectional studies. ${ }^{a}$ Analysis was adjusted for age, hypertension, smoking status, dyslipidemia, BMI, waist circumstance, CRP, Agatston score, and ABI. ${ }^{b}$ Analysis was adjusted for age, sex, race, education, marital status, smoking, coffee and alcohol consumption, calorie intake, family history of diabetes, general health status, nocturnal sleep duration, physical activity, and BMI. ${ }^{c}$ Analysis was adjusted for age, sex, marriage, education, smoking status, alcohol intake, hypertension, coronary heart disease, stroke, nocturnal sleep duration, BMI, and physical activity. ${ }^{\mathrm{d}}$ Analysis was adjusted for age, sex, educational level, occupation, smoking, alcohol intake, physical activity, health status (self-rated health, hospitalization, hypertension, cardiovascular disease, and family history of diabetes), adiposity and metabolic markers (waist circumference, triglycerides, and total cholesterol), and sleep variables (total sleep duration, insomnia, daytime sleepiness, and snoring). The estimate for the relation between a short nap time and diabetes was calculated from a subsample $(n=3,822)$ of the much larger study population $(n=19,567) .{ }^{e}$ Analysis was stratified by presence of snoring. ${ }^{\mathrm{f}}$ Analysis was adjusted for age, BMI, alcohol dependency, level of physical activity, and smoking status. ${ }^{\mathrm{g}}$ Analysis was adjusted for age, sex, BMI, depression, smoking, alcohol use, allergy, asthma, hypertension, thyroid function, night time sleep duration, and objective polysomnographic data. ${ }^{\mathrm{h}}$ Analysis was adjusted for sex, depression, use of sleeping medication, smoking, and BMI. ${ }^{i}$ Analysis was adjusted for age, general health, frequent awakening, ability to fall asleep again after nocturnal awakening, and hypnotic medication. ${ }^{j}$ Analysis was adjusted for age, marriage, education, smoking status, drinking status, physical activity, coronary heart disease, myocardial infarction, stroke, family history of hypertension and diabetes, BMI, and nighttime sleep duration. ${ }^{\mathrm{A}}$ Analysis was adjusted for age, BMI, current smoking, drinking status, physical activity, and sleeping hours (total nocturnal sleeping hours). 
Association of daytime napping with the risk of type 2 diabetes.

a. Long Nap ( $\geq 60 \mathrm{~min} /$ day) vs. No Nap
Study
Fang
Xu

b. Short Nap (<60 min/day) vs. No Nap

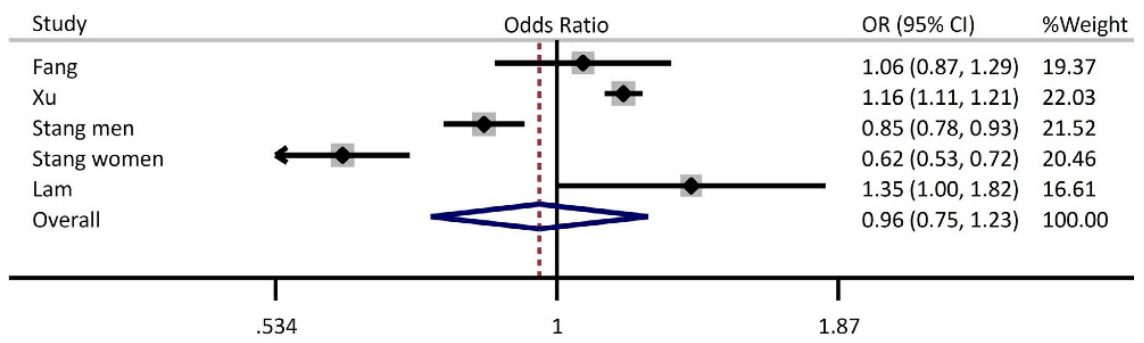

Figure 2. Association of daytime napping with the risk of type 2 diabetes. Plots show the association between daytime napping and the risk of diabetes. $\mathrm{CI}=$ confidence interval. $\mathrm{OR}=$ odds ratio.

\section{Meta-analysis of the odds ratio of developing diabetes}

\begin{tabular}{|c|c|c|c|c|c|c|}
\hline & No. of reports & Odds ratio & Odds ratio $(95 \% \mathrm{Cl})$ & $P$ for test & $I^{2}(\%)$ & $P$ for heterogeneity \\
\hline \multicolumn{7}{|l|}{ Long nap (vs. no nap) } \\
\hline Total & 5 & & $1.46(1.23-1.74)$ & $<0.001$ & 89 & $<0.001$ \\
\hline \multicolumn{7}{|l|}{ Study location } \\
\hline Western & 3 & $\longrightarrow$ & $1.82(1.23-2.71)$ & $<0.001$ & 91 & $<0.001$ \\
\hline Asian & 2 & $\rightarrow$ & $1.2(1.07-1.34)$ & 0.02 & 57 & 0.13 \\
\hline \multicolumn{7}{|l|}{ Study quality } \\
\hline New castle Ottawa scale 8 & 1 & $\rightarrow$ & $1.28(1.14-1.44)$ & $<0.001$ & - & - \\
\hline New castle Ottawa scale 7 & 4 & $\longrightarrow$ & $1.55(1.23-1.96)$ & $<0.001$ & 92 & $<0.001$ \\
\hline \multicolumn{7}{|l|}{ Study type } \\
\hline Cross-sectional study & 4 & $\longrightarrow$ & $1.55(1.19-2.03)$ & 0.001 & 91 & $<0.001$ \\
\hline Cohort study & 1 & 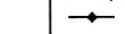 & $1.36(1.27-1.46)$ & $<0.001$ & - & - \\
\hline \multicolumn{7}{|l|}{ Short nap (vs. no nap) } \\
\hline Total & 5 & 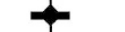 & $0.96(0.75-1.23)$ & 0.75 & 96 & $<0.001$ \\
\hline \multicolumn{7}{|l|}{ Study location } \\
\hline Western & 3 & - & $0.85(0.61-1.19)$ & 0.35 & 98 & $<0.001$ \\
\hline Asian & 2 & + & $1.16(0.92-1.47)$ & 0.2 & 43 & 0.19 \\
\hline \multicolumn{7}{|l|}{ Study quality } \\
\hline New castle Ottawa scale 8 & 1 & $\infty$ & $1.35(1.00-1.82)$ & 0.05 & - & - \\
\hline New castle Ottawa scale 7 & 4 & - & $0.9(0.69-1.18)$ & 0.44 & 97 & $<0.001$ \\
\hline \multicolumn{7}{|l|}{ Study type } \\
\hline Cross-sectional study & 4 & $\leftarrow$ & $0.91(0.70-1.18)$ & 0.48 & 90 & $<0.001$ \\
\hline Cohort study & 1 & $\rightarrow$ & $1.16(1.11-1.21)$ & $<0.001$ & - & - \\
\hline
\end{tabular}

Figure 3. Meta-analysis of the odds ratio of developing diabetes. Plots show the association between daytime napping and the risk of diabetes. $\mathrm{CI}=$ confidence interval. $\mathrm{OR}=$ odds ratio.

relevant local rules for ethics and data protection. Studies that did not report risks independently stratified by at least 3 nap time categories (e.g., $0 \mathrm{~min},<60 \mathrm{~min}$, and $>60 \mathrm{~min}$ per day) were excluded from this meta-analysis.

Data Extraction and Assessment of Study Quality. We extracted information on the characteristics of each study (study name, authors, year of publication, journal, study type, study location, and number of participants and incident cases), the subject characteristics (age, sex, and BMI), the extent of exposure to napping (definition of napping, nap time, and prevalence of napping in each category), the validity of the method used 
Association of excessive daytime sleepiness with the risk of type 2 diabetes.

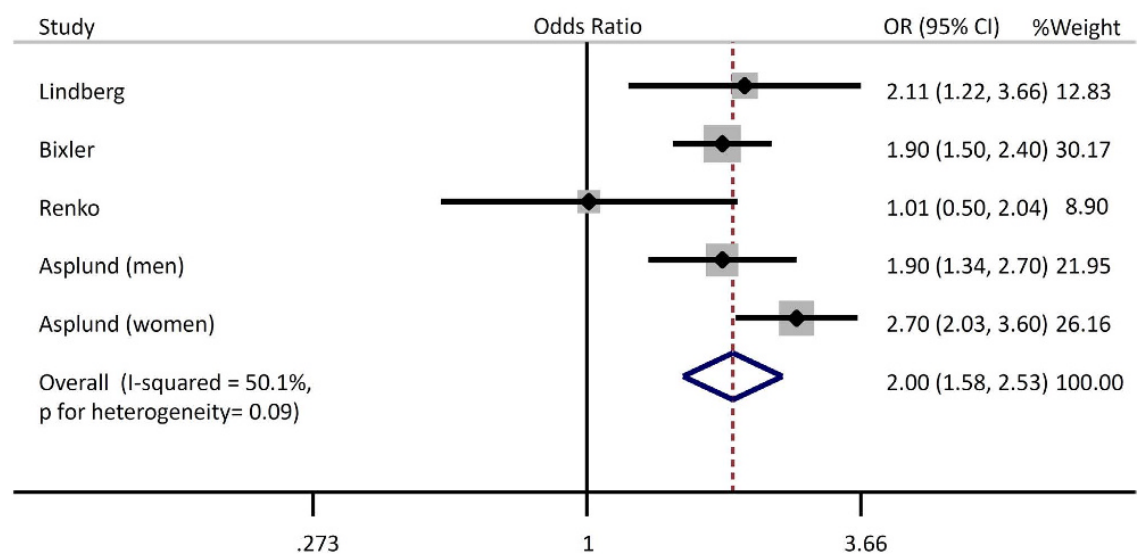

Figure 4. Association of excessive daytime sleepiness with the risk of type 2 diabetes. Plots show the association between excessive daytime sleepiness and the risk of diabetes. $\mathrm{CI}=$ confidence interval. $\mathrm{OR}=\mathrm{odds}$ ratio.

Dose-response relationship between nap time and the risk of type 2 diabetes

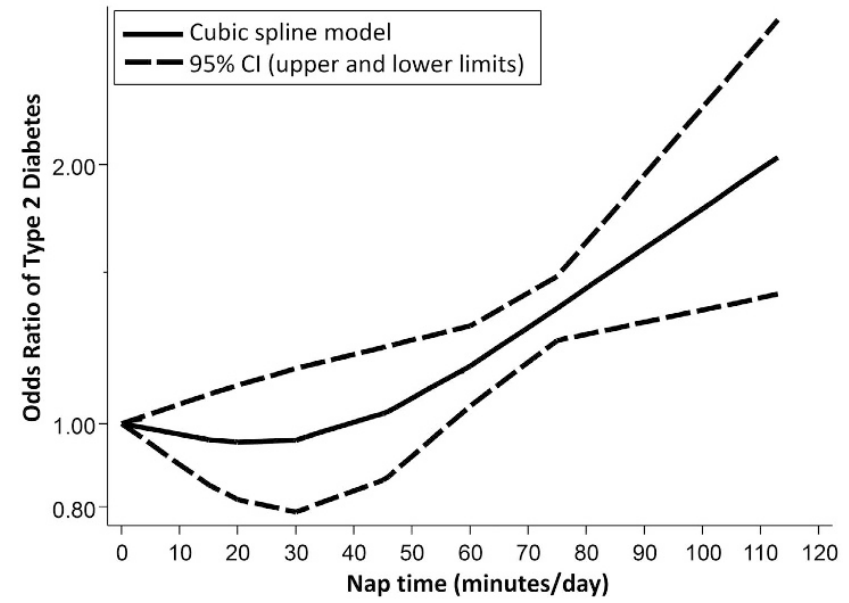

Figure 5. Dose-response relationship between nap time and the risk of type 2 diabetes. $\mathrm{CI}=$ confidence interval.

for assessment of napping (and excessive daytime sleepiness), the validity of the method used for assessment of the outcome (diabetes and metabolic syndrome), and the validity of the analytical methods (statistical models, covariates included in the models, and risk estimates for each nap duration category). Some studies reported data separately for men and women, so we treated each of these cohorts as an independent report and extracted data separately. If more than one study covered the same cohort, only the report containing the most comprehensive information was used to avoid analysis of overlapping populations.

To ascertain the validity of the studies, the quality of each report was appraised with reference to the STROBE statement ${ }^{25}$. In addition, the Newcastle-Ottawa Scale for assessing the quality of observational studies in meta-analyses was used to quantify the validity of each study ${ }^{26}$. Only high-quality observational studies with a Newcastle-Ottawa Scale score $\geq 6$ (maximum possible score: 9) were included in this meta-analysis. Two authors (T Yamada and NS) independently confirmed the eligibility of the studies and then extracted and collated the data. Any discrepancies were resolved through discussion.

Statistical analysis. The pooled odds ratio (OR) and its $95 \%$ confidence interval (CI) was employed to assess associations in all of the studies, except for use of the prevalence ratio by Stang et al. ${ }^{15}$. Because the incidence of events was not high in their study, the prevalence ratio was considered to be a relatively accurate estimate of the true OR. We pooled all odds ratios by using the DerSimonian-Laird random effects model to compare 
Association of daytime napping with the risk of metabolic syndrome.

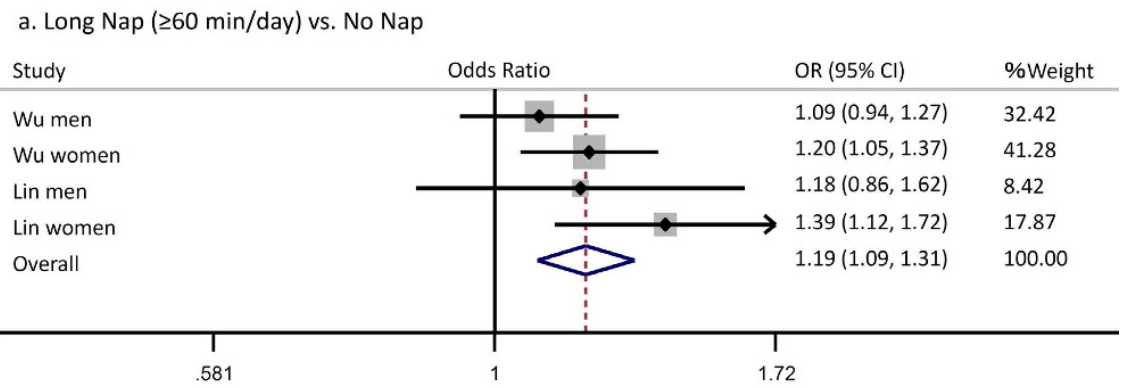

b. Short Nap (<60 min/day) vs. No Nap

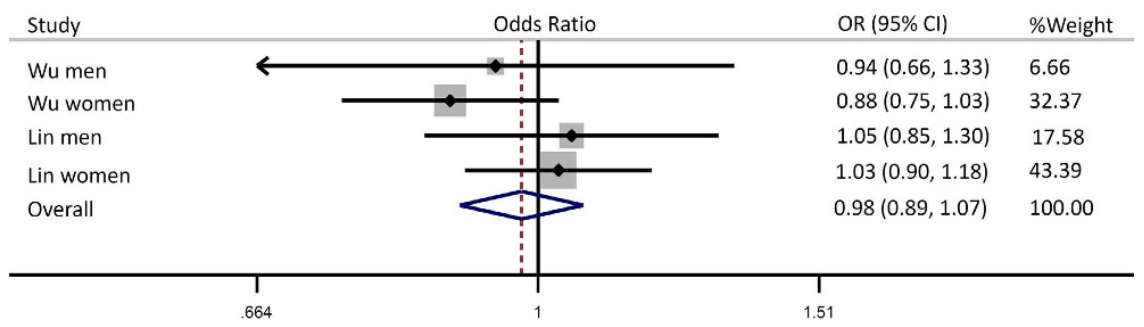

Figure 6. Association of daytime napping with the risk of metabolic syndrome. Plots show the association between daytime napping and the risk of diabetes. $\mathrm{CI}=$ confidence interval. $\mathrm{OR}=$ odds ratio.

napping categories and set study weights as equal to the inverse variance of the estimated effect for each study ${ }^{27}$. Cochrane's $\chi^{2}$ test and the $I^{2}$ test were used to evaluate heterogeneity among the studies ${ }^{28}$.

We included the odds ratios of the longest (or shortest) groups in the pairwise meta-analysis (such as long nap vs. no nap, short nap vs. no nap) if the published studies reported odds ratios for various nap times (e.g., 60 to $<90$ minutes vs. $>90$ minutes). Stratified analyses were also performed with stratification by study location, study score, and study type, and we used the method of Altman et al. ${ }^{29}$ to evaluate whether the pooled ORs differed between groups stratified by the study location. In the study of Stang et al. ${ }^{15}$, the prevalence ratio for napping and diabetes was standardized by age, but was not adjusted for other potential confounders. Therefore, we performed a sensitivity analysis that excluded this study and investigated whether there was any change of the point estimate.

Possible publication bias was evaluated by creating a funnel plot of the effect size for each study versus the standard error. Then asymmetry of the funnel plots was assessed by performing Begg's test ${ }^{30}$ and Egger's test ${ }^{31}$. To evaluate the potential dose-response relation between diabetes and nap time, a dose-response meta-analysis was performed taking into account the between-study heterogeneity proposed by Orsini et al. ${ }^{32}$ to compute the trend from correlated log values of OR estimates across various nap times. A restricted cubic spline model for the duration of nap time with three knots (5th, 35 th, 65 th, and 95 th percentiles) ${ }^{33}$ was estimated by generalized least squares regression analysis, taking into account the correlations within each set of published ORs ${ }^{34}$. Probability (P) values for curve linearity or non-linearity were calculated by testing the null hypothesis that the coefficient of the second spline equals zero. This analysis incorporated data on the ORs and 95\% CIs, the number of cases and participants, and the median or mean nap time (minutes per day) for each group.

The midpoint of the upper and lower borders was set as the median dose for each category if the median or mean exposure per category was not reported. If the highest category was open-ended, the midpoint of the category was set at 1.25 times the lower border. For the lowest nap category, we set the median at 0.5 times the cut-off point (e.g., if category was $<30 \mathrm{~min}$, the median was set at $15 \mathrm{~min}$ ). In the study of Lam et al. ${ }^{18}$, we set the median time for "napping 1-3 times per week" at $20 \mathrm{~min}$ and the median time for "napping 4-6 times per week" at $45 \mathrm{~min}$, taking into account the fact that the mean nap time in this study was $60 \mathrm{~min} / \mathrm{day}$.

Because the estimators in the random effects cubic spline model were found to be unstable due to lack of power, we used a fixed effects model to evaluate the dose-response relation. All statistical analyses were performed with Stata V.14.0 software (StataCorp, College Station, Texas, USA). P values of less than 0.05 were considered significant.

This research was carried out according to a predetermined protocol ${ }^{35}$ (Table S2), and it followed the standard guidelines for the conduct and reporting of systematic reviews and network meta-analyses of the Meta-analysis Of Observational Studies in Epidemiology (MOOSE) group ${ }^{36}$ (Checklist S1) and the PRISMA statement ${ }^{37}$ (Checklist S2).

\section{Results}

Literature search. Figure 1 summarizes the processes employed for literature search and study selection. We identified 965 articles from electronic databases and other sources. After excluding 917 studies that did not meet our inclusion criteria, 48 articles were subjected to full-text evaluation. Finally, 10 studies were included in the meta-analysis ${ }^{15-24}$. A manual search of the references cited in the reports on these studies did not yield any 
Dose-response relationship between nap time and the risk of metabolic syndrome

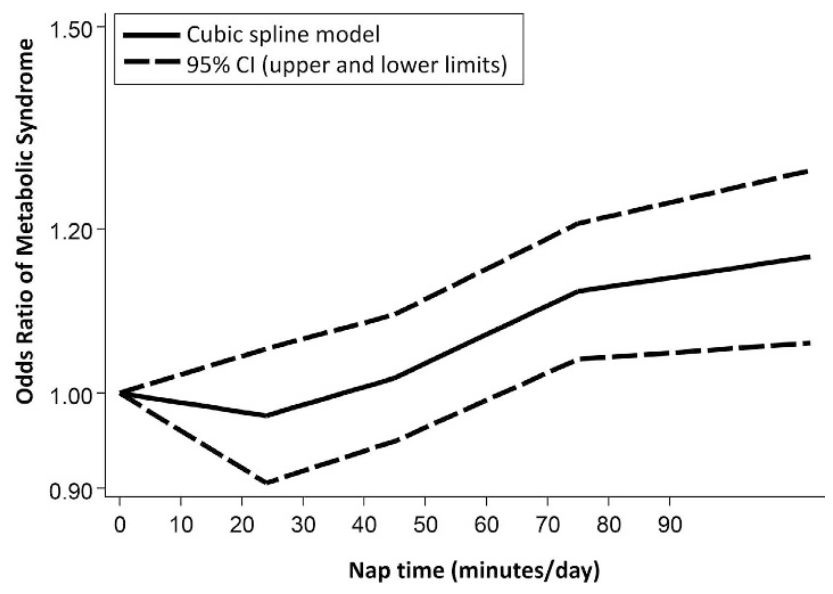

Figure 7. Dose-response relationship between nap time and the risk of metabolic syndrome. $\mathrm{CI}=$ confidence interval.

new eligible articles for assessment. Four of the studies ${ }^{15,19,20,24}$ examined men and women separately, so the data on men and women from each of these studies were handled as if from separate reports. In addition, Lindberg et al. ${ }^{21}$ analysed data separately based on the presence of snoring, but we only included the ORs for EDS and diabetes from the EDS without snoring group in the meta-analysis, because the ORs of the two subgroups (EDS with snoring and EDS without snoring subgroups) overlapped. Finally, data on a total of 14 distinct cohorts were included in the meta-analysis.

Study characteristics. Table 1 lists the characteristics of the studies included in the present analysis, which covered 288,883 subjects from Western and Asian populations, including 20,109 patients with diabetes and 11,222 patients with metabolic syndrome. The mean age, prevalence of napping, and EDS were largely in the range between $60-65$ years, $50-70 \%$, and $10-20 \%$, respectively. Four studies were conducted in China ${ }^{17-20}$, followed by two each in Sweden ${ }^{21,24}$, and one each in the USA, Spain, Germany and Finland. In most studies, the analyses were well adjusted for several confounders related to the risk of diabetes and were also adjusted for sleep parameters (such as nocturnal sleep duration). In the study of Lam et al. ${ }^{18}$, the estimated relation between a short nap and diabetes was calculated from a subsample $(\mathrm{n}=3.822)$ of the much larger study population $(\mathrm{n}=19.567)$. The definitions of napping and EDS were similar in all of the studies. Study subjects were generally classified as having diabetes if they reported a diagnosis of diabetes made by a physician, were on antidiabetic medication, or had a high fasting plasma glucose level ( $\geq 7.0 \mathrm{mmol} / \mathrm{L})$ (Table S3). Subjects were classified as having metabolic syndrome if they fulfilled the criteria of the International Diabetes Federation (IDF) ${ }^{38}$. When the quality of the studies was assessed by using the Newcastle Ottawa Scale, all studies had high scores $\geq 7$ (maximum possible score: 9) (Table S4).

Results of meta-analysis. Napping and type 2 diabetes. Figure 2 summarizes data on the random effects odds ratio $(95 \% \mathrm{CI})$ of diabetes. Pooled analysis revealed that a longer nap time ( $\geq 60 \mathrm{~min} /$ day $)$ was associated with a significantly higher risk of type 2 diabetes (odds ratio: 1.46 [95\% confidence interval: 1.23-1.74, $p<0.01$, $\left.\left.I^{2}=89 \%\right]\right)$ compared with the absence of these factors, while a shorter nap time $(<60 \mathrm{~min} /$ day $)$ was not associated with an increased risk of diabetes (odds ratio $0.96\left[0.75-1.23, p=0.75, I^{2}=96 \%\right]$ ). Similar results were obtained when analyses were performed with stratification by study location, study quality, and study type (Fig. 3). Heterogeneity showed a slight decrease in the analysis stratified by study location (Western or Asian) without any significant interaction ( $\mathrm{p}$ for interaction $=0.05$ for long nap vs. no nap, $\mathrm{p}$ for interaction $=0.14$ for short nap vs. no nap). Moreover, a sensitivity analysis excluding the study of Stang et al. ${ }^{15}$, in which the prevalence ratio for napping and diabetes was standardized by age but not adjusted for other potential confounders, also yielded a similar result (OR1.26 [1.13-1.41, $p<0.01, I^{2}=77 \%$ ) (long nap vs. no nap). The funnel plot, Begg's test, and Egger's test did not suggest any evidence of publication bias (Figure S1). In addition, we found that excessive daytime sleepiness was also associated with an increased risk of type 2 diabetes (odds ratio 2.00 [1.58-2.53, $p<0.01$, $\left.I^{2}=50 \%\right]$ ) (Fig. 4).

The fixed effects cubic spline model incorporated a total of 208,226 subjects, including 15,571 patients with diabetes. Raw data were available for 5 distinct comparisons with a total of $18 \mathrm{log}$ odds ratios. Cubic spline meta-analysis revealed that there was a J-curve dose-response relation between nap time and the risk of type 2 diabetes $(P$ for non-linearity $=0.003)($ Fig. 5$)$. That is, the odds ratio of developing type 2 diabetes initially decreased at nap times from 0 to $30 \mathrm{~min} /$ day. Then the risk began to increase, but prolongation of the nap time had little effect up to about $40 \mathrm{~min} /$ day, followed by a sharp increase in the risk of diabetes at longer times. Napping for 90 minutes increased the risk of developing diabetes by up to $50 \%$. 
Napping and metabolic syndrome. Pooled analysis revealed that a longer nap time ( $\geq 60 \mathrm{~min} /$ day) was associated with a significantly higher risk of metabolic syndrome (odds ratio: 1.19 [95\% confidence interval: 1.09-1.31, $\left.\left.p<0.01, I^{2}=10 \%\right]\right)$, while a shorter nap time ( $<60 \mathrm{~min} /$ day $)$ was not associated with an increased risk of metabolic syndrome (odds ratio $0.98\left[0.89-1.07, p=0.6, I^{2}=0 \%\right]$ ) (Fig. 6). Similar to diabetes, the relationship between napping and metabolic syndrome fitted a J-shaped curve (Fig. 7). Napping for less than $40 \mathrm{~min} /$ day was not associated with an increased risk of metabolic syndrome, but the risk increased sharply at nap times beyond $40 \mathrm{~min}$. In addition, the funnel plot, Begg's test, and Egger's test did not suggest any evidence of publication bias (data not shown).

\section{Discussion}

This meta-analysis showed that either a long nap or EDS was associated with an increased risk of type 2 diabetes and metabolic syndrome compared with not napping or no EDS. In contrast, a short nap was not associated with diabetes or metabolic syndrome. We also demonstrated that nap time may show a J-curve relationship with these metabolic diseases, as has been reported for cardiovascular disease $\mathrm{e}^{14}$.

As we have stated ${ }^{14}$, it is possible that longer reactive naps are associated with unfavorable outcomes such as poor health, pain, and depression ${ }^{24}$, while older men with sleep apnea may be more likely to take longer naps to compensate for fragmented nocturnal sleep ${ }^{8}$. Several studies have shown that patients with obstructive sleep apnea have increased glucose levels, increased insulin resistance, and a higher risk of type 2 diabetes and metabolic syndrome $\mathrm{e}^{2439,40}$. Although previous studies that involved meta-analysis were well adjusted for the duration of nocturnal sleep (i.e., the quantity of sleep), the quality of sleep was not well adjusted, so the association that we noted between a longer nap time and an increased risk of metabolic diseases might have arisen due to impairment of sleep quality by obstructive sleep apnea or other factors.

Moreover, a shorter nap might not have the same negative effects as a longer nap. Several studies have demonstrated that a daytime nap of less than $30 \mathrm{~min}$ promotes wakefulness and alertness, reduces sleep deficits, and enhances performance and learning ${ }^{41-45}$. These multiple benefits, especially enhanced athletic performance, might be associated with the lower risk of diabetes for persons taking a short nap in our study. A possible physiological basis for the differing influence of short versus long naps is the relation between nap time and the sleep cycle. A short nap would be expected to end before the onset of deep slow-wave sleep, while a long nap would finish during the slow-wave sleep period. It is known that failing to complete the normal sleep cycle after entering slow-wave sleep can result in a phenomenon known as sleep inertia, which leaves a person feeling groggy, disoriented, and even sleepier than before napping ${ }^{11,46}$.

Intermittent hypoxemia and sleep fragmentation increase sympathetic activity ${ }^{47}$, and increased sympathetic activity impairs glucose homeostasis by enhancing the breakdown of glycogen and gluconeogenesis ${ }^{48}$. Alterations of neuroendocrine function and release of proinflammatory mediators (e.g., tumor necrosis factor- $\alpha$ and interleukin-6) may also occur along with autonomic activation ${ }^{49-51}$. Although the mechanisms underlying the beneficial effect of a short nap are still unclear, it might modify these endocrine abnormalities caused by sleep deprivation and improve the circadian rhythm.

Our present meta-analysis had some limitations. First and importantly, while we analysed results from adjusted models because the original studies were observational investigations, this meta-analysis was mainly based on case-control studies and thus cannot prove causal effects. Moreover, the findings could have been influenced by residual confounders or other biases (habits and frailty, health worker bias, fatal malignancy, funding source, etc.). Thus, the associations detected in the individual studies and our meta-analysis may have been biased in either direction. Second, a high level of heterogeneity was detected. To explore the reasons for this, we performed analyses stratified by study location, study quality, and study type. However, heterogeneity was not dramatically reduced by these stratified analyses. Because we identified a nonlinear J-curve relation between nap time and diabetes or metabolic syndrome in our dose-response meta-analysis, it is possible that differences of nap time among the studies analyzed might have led to high heterogeneity. Third, there was limited information about the possible contribution of physical activity to diabetes. Leng et al. reported that inactive people have a higher prevalence of napping compared with active people ${ }^{52}$, so the level of activity might be an important confounder.

Ideally, our results should be confirmed by prospective clinical trials, but it seems difficult to perform an interventional longitudinal study of napping in the real world (participants would often be unable to nap for the specified time). Accordingly, we cannot rule out the possibility of "post hoc ergo propter hoc".

Fourth, we might have overlooked some relevant articles, which could have led to selection bias. However, we investigated all of the references cited in each study we selected to avoid this risk as far as possible. Also, our analyses of publication bias did not suggest that unpublished results had been missed. Fifth, all of the studies used structured interviews or self-completed questionnaires to assess nap times, so measurement error is inevitable. However, measurement error with regard to assessment of exposure is more likely to cause attenuation of a true association than to exaggerate a weak association. Sixth, although our funnel plot analyses did not show significant publication bias, the limited number of studies may have diminished the statistical power for detecting heterogeneity. Finally, we employed different measures ("standardized increment of nap time" or midpoint of nap time) when modeling the associations of the dose-response relation in our meta-analysis. However, none of the studies gave precise data on nap times, which raises concern regarding the precision of our analyses. Despite these limitations, we consider that our meta-analysis provides useful evidence regarding the potential influence of napping on metabolic disease.

In conclusion, the relation between nap time and the risk of diabetes or metabolic syndrome seems to be described by a J-curve, with a sharp increase in the risk of diabetes or metabolic syndrome at longer nap times. In contrast, a short nap was not associated with an increased risk of either diabetes or metabolic syndrome. 
Additional research, including large-scale pooling projects, with accurate and detailed measures of napping will be needed to confirm our conclusions and to determine whether adding a nap time score to a conventional risk model improves estimation of the long-term risk of diabetes in the general population, as well as whether a short nap decreases the risk of developing type 2 diabetes.

\section{References}

1. Ohkuma, T. et al. Impact of sleep duration on obesity and the glycemic level in patients with type 2 diabetes: the Fukuoka Diabetes Registry. Diabetes Care. 36(3), 611-7 (2013).

2. Cappuccio, F. P., D’Elia, L., Strazzullo, P. \& Miller, M. A. Quantity and quality of sleep and incidence of type 2 diabetes: a systematic review and meta-analysis. Diabetes Care. 33, 414-420 (2010).

3. Cappuccio, F. P., Cooper, D., D’Elia, L., Strazzullo, P. \& Miller, M. A. Sleep duration predicts cardiovascular outcomes: a systematic review and meta-analysis of prospective studies. Eur Heart J. 32(12), 1484-92 (2011).

4. Leng, Y. et al. Sleep duration and risk of fatal and nonfatal stroke: a prospective study and meta-analysis. Neurology. 84(11), 1072-9 (2015).

5. Tamakoshi, A. \& Ohno, Y. Self-reported sleep duration as a predictor of all-cause mortality: results from the JACC study, Japan. Sleep. 27, 51-54 (2004)

6. Shen, X., Wu, Y. \& Zhang, D. Nighttime sleep duration, 24-hour sleep duration and risk of all-cause mortality among adults: a metaanalysis of prospective cohort studies. Sci Rep. 22, 21480 (2016).

7. Liu, Y., Wheaton, A. G., Chapman, D. P., Cunningham, T. J., Lu, H. \& Croft, J. B. Prevalence of Healthy Sleep Duration among Adults - United States, 2014. Centers for Disease Control and Prevention: CDC. Available at: http://www.cdc.gov/mmwr/volumes/65/wr/ mm6506a1.htm (accessed: 24th April 2016) (2014).

8. Guilleminault, C., Stoohs, R., Clerk, A., Cetel, M. \& Maistros, P. A cause of excessive daytime sleepiness. The upper airway resistance syndrome. Chest. 104, 781-787 (1993).

9. Stang, A. et al. Heinz Nixdorf Recall Investigative Group. Midday naps and the risk of coronary artery disease: results of the Heinz Nixdorf Recall Study. Sleep. 35(12), 1705-12 (2012).

10. Jung, K. I., Song, C. H., Ancoli-Israel, S. \& Barrett-Connor, E. Gender differences in nighttime sleep and daytime napping as predictors of mortality in older adults: the Rancho Bernardo study. Sleep Med. 14(1), 12-9 (2013).

11. Dhand, R. \& Sohal. Good sleep, bad sleep! The role of daytime naps in healthy adults. Curr Opin Pulm Med. 12, 379-382 (2006)

12. Naska, A., Oikonomou, E., Trichopoulou, A., Psaltopoulou, T. \& Trichopoulos, D. Siesta in healthy adults and coronary mortality in the general population. Arch Intern Med. 167, 296-301 (2007).

13. Asada, T., Motonaga, T., Yamagata, Z., Uno, M. \& Takahashi, K. Associations between retrospectively recalled napping behavior and later development of Alzheimer's disease: association with APOE genotypes. Sleep. 23(5), 629-34 (2000).

14. Yamada, T., Hara, K., Shojima, N., Yamauchi, T. \& Kadowaki, T. Daytime Napping and the Risk of Cardiovascular Disease and AllCause Mortality: A Prospective Study and Dose-Response Meta-Analysis. Sleep. 38(12), 1945-53 (2015).

15. Stang, A. et al. Daily siesta, cardiovascular risk factors, and measures of subclinical atherosclerosis: results of the Heinz Nixdorf Recall Study. Sleep. 30(9), 1111-9 (2007).

16. Xu, Q. et al. Day napping and short night sleeping are associated with higher risk of diabetes in older adults. Diabetes Care. 33(1), 78-83 (2010).

17. Fang, W. et al. Longer habitual afternoon napping is associated with a higher risk for impaired fasting plasma glucose and diabetes mellitus in older adults: results from the Dongfeng-Tongji cohort of retired workers. Sleep Med. 14(10), 950-4 (2013).

18. Lam, K. B. et al. Napping is associated with increased risk of type 2 diabetes: the Guangzhou Biobank Cohort Study. Sleep. 33(3), 402-7 (2010)

19. Wu, J. et al. Daily sleep duration and risk of metabolic syndrome among middle-aged and older Chinese adults: cross-sectional evidence from the Dongfeng-Tongji cohort study. BMC Public Health. 15, 178 (2015).

20. Lin, D. et al. Association between habitual daytime napping and metabolic syndrome: a population-based study. Metabolism. 63(12), 1520-7 (2014).

21. Lindberg, E., Berne, C., Franklin, K. A., Svensson, M. \& Janson, C. Snoring and daytime sleepiness as risk factors for hypertension and diabetes in women - a population-based study. Respir Med. 101(6), 1283-90 (2007).

22. Bixler, E. O. et al. Excessive daytime sleepiness in a general population sample: the role of sleep apnea, age, obesity, diabetes, and depression. J Clin Endocrinol Metab. 90(8), 4510-5 (2005).

23. Renko, A. K., Hiltunen, L., Laakso, M., Rajala, U. \& Keinänen-Kiukaanniemi, S. The relationship of glucose tolerance to sleep disorders and daytime sleepiness. Diabetes Res Clin Pract. 67(1), 84-91 (2005).

24. Asplund, R. Daytime sleepiness and napping amongst the elderly in relation to somatic health and medical treatment. J Intern Med. 239(3), 261-7 (1996).

25. von Elm, E. et al. STROBE Initiative. The Strengthening the Reporting of Observational Studies in Epidemiology (STROBE) statement: guidelines for reporting observational studies. J Clin Epidemiol. 61(4), 344-9 (2008).

26. Wells, G. et al. The Newcastle-Ottawa Scale (NOS) for assessing the quality of nonrandomised studies in meta-analyses. Ottawa Hospital Research Institute. Available at: http://www.ohri.ca/programs/clinical_epidemiology/oxford.asp (Accessed 24th April 2016).

27. DerSimonian, R. \& Laird, N. Meta-analysis in clinical trials. Control Clin Trials. 7(3), 177-88 (1986).

28. Higgins, J. P. \& Thompson, S. G. Quantifying heterogeneity in a meta-analysis. Stat Med. 21(11), 1539-58 (2002)

29. Altman, D. G. \& Bland, J. M. Interaction revisited: the difference between two estimates. BMJ. 326, 219 (2003).

30. Begg, C. B. \& Mazumdar, M. Operating characteristics of a rank correlation test for publication bias. Biometrics. 50(4), 1088-101 (1994).

31. Egger, M., Davey Smith, G., Schneider, M. \& Minder, C. Bias in meta-analysis detected by a simple, graphical test. BMJ. 315, 629-34 (1997).

32. Orsini, N., Li, R., Wolk, A., Khudyakov, P. \& Spiegelman, D. Meta-analysis for linear and nonlinear dose-response relations: examples, an evaluation of approximations, and software. Am J Epidemiol. 175(1), 66-73 (2012).

33. Harrell, F. E. Jr., Lee, K. L. \& Pollock, B. G. Regression models in clinical studies: determining relationships between predictors and response. J Natl Cancer Inst. 80(15), 1198-202 (1988).

34. Orsini, N., Bellocco, R. \& Greenland, S. Generalized least squares for trend estimation of summarized dose-response data. Stata J. 6(1), 40-57 (2006).

35. Shamseer, L. et al. PRISMA-P Group. Preferred reporting items for systematic review and meta-analysis protocols (PRISMA-P) 2015: elaboration and explanation. BMJ. 349, g7647 (2015).

36. Stroup, D. F. et al. Meta-analysis of observational studies in epidemiology: a proposal for reporting. Meta-analysis Of Observational Studies in Epidemiology (MOOSE) group. JAMA. 283(15), 2008-12 (2000).

37. Liberati, A. et al. The PRISMA statement for reporting systematic reviews and meta-analyses of studies that evaluate health care interventions: explanation and elaboration. Ann Intern Med. 151(4), W65-94 (2009). 
38. Alberti, K. G., Zimmet, P. \& Shaw, J. IDF Epidemiology Task Force Consensus Group. The metabolic syndrome-a new worldwide definition. Lancet. 366(9491), 1059-62 (2005).

39. Zizi, F. et al. Sleep duration and the risk of diabetes mellitus: epidemiologic evidence and pathophysiologic insights. Curr Diab Rep. 10(1), 43-7 (2010).

40. Spiegel, K., Knutson, K., Leproult, R., Tasali, E. \& Van Cauter, E. Sleep loss: a novel risk factor for insulin resistance and type II diabetes. J Appl Physiol. 99, 2008-2019 (2005).

41. Gillberg, M., Kecklund, G., Axelsson, J. \& Akerstedt, T. The effects of a short daytime nap after restricted night sleep. Sleep. 19(7), $570-5(1996)$

42. Hayashi, M., Ito, S. \& Hori, T. The effects of a 20 -min nap at noon on sleepiness, performance and EEG activity. Int J Psychophysiol. 32(2), 173-80 (1999).

43. Hayashi, M., Watanabe, M. \& Hori, T. The effects of a 20 min nap in the mid-afternoon on mood, performance and EEG activity. Clin Neurophysiol. 110(2), 272-9 (1999).

44. Takahashi, M. \& Arito, H. Maintenance of alertness and performance by a brief nap after lunch under prior sleep deficit. Sleep. 23(6), $813-9(2000)$.

45. Tietzel, A. J. \& Lack, L. C. The short-term benefits of brief and long naps following nocturnal sleep restriction. Sleep. 24(3), 293-300 (2001).

46. Milner, C. E. \& Cote, K. A. Benefits of napping in healthy adults: impact of nap length, time of day, age, and experience with napping. J Sleep Res. 18, 272-281 (2009).

47. Somers, V. K., Dyken, M. E., Clary, M. P. \& Abboud, F. M. Sympathetic neural mechanisms in obstructive sleep apnea. J Clin Invest. 96, 1897-1904 (1995).

48. Punjabi, N. M., Ahmed, M. M., Polotsky, V. Y., Beamer, B. A. \& O’Donnell, C. P. Sleep-disordered breathing, glucose intolerance, and insulin resistance. Respir Physiol Neurobiol. 136, 167-178 (2003).

49. Tasali, E., Mokhlesi, B. \& Van Cauter, E. Obstructive sleep apnea and type 2 diabetes: interacting epidemics. Chest. 133(2), 496-506 (2008).

50. Punjabi, N. M. \& Polotsky, V. Y. Disorders of glucose metabolism in sleep apnea. Association of sleep time with diabetes mellitus and impaired glucose tolerance. J Appl Physiol. 99, 1998-2007 (2005).

51. Punjabi, N. M. et al.: Sleep-disordered breathing and insulin resistance in middle-aged and overweight men. Am J Respir Crit Care Med. 165, 677-682 (2002).

52. Leng, Y. et al. Daytime napping, sleep duration and increased 8-year risk of type 2 diabetes in a British population. Nutr Metab Cardiovasc Dis. Jun 28. pii: S0939-4753(16)30086-2 (2016).

\section{Acknowledgements}

T Yamada was funded by Japan Diabetes Society, Banyu Life Science Foundation International, the Grant-in-Aid for Young Scientists (B)(16K20965) from the Japan Society for the Promotion of Science, Japan Foundation for Applied Enzymology, and Grants for young researchers from Japan Association for Diabetes Education and Care.

\section{Author Contributions}

T. Yamada, N.S., T. Yamauchi and T.K. conceived the concept of the study and were responsible for its design. T. Yamada and N.S. were responsible for acquisition of the data. T. Yamada and N.S. were responsible for undertaking data analysis and produced the figures. T. Yamada, N.S., T. Yamauchi, and T.K. contributed to interpretation of the results. The initial draft of the manuscript was prepared by T. Yamada and N.S. and then was circulated repeatedly among all authors for critical revision.

\section{Additional Information}

Supplementary information accompanies this paper at http://www.nature.com/srep

Competing financial interests: The authors declare no competing financial interests.

How to cite this article: Yamada, T. et al. J-curve relation between daytime nap duration and type 2 diabetes or metabolic syndrome: A dose-response meta-analysis. Sci. Rep. 6, 38075; doi: 10.1038/srep38075 (2016).

Publisher's note: Springer Nature remains neutral with regard to jurisdictional claims in published maps and institutional affiliations.

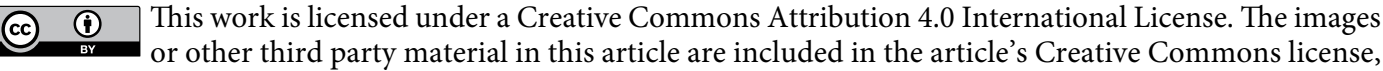
unless indicated otherwise in the credit line; if the material is not included under the Creative Commons license, users will need to obtain permission from the license holder to reproduce the material. To view a copy of this license, visit http://creativecommons.org/licenses/by/4.0/

(c) The Author(s) 2016 